\title{
Genotype Summary
}

National Cancer Institute

\section{Source}

National Cancer Institute. Genotype Summary. NCI Thesaurus. Code C41098.

Listing of the genetic changes made deliberately in order to generate the animal model. 